\title{
Does Rapid Eye Movement Sleep Aggravate Obstructive Sleep Apnea?
}

\author{
Sung Hee Kim ${ }^{1, \star}$ () $\cdot$ Chan Joo Yang ${ }^{2, \star} \cdot$ Jong Tae Baek ${ }^{1} \cdot$ Sang Min Hyun ${ }^{3} \cdot$ Cheon Sik Kim ${ }^{4}$ Sang-Ahm Lee \\ Yoo-Sam Chung ${ }^{5}$ (D) \\ ${ }^{I}$ Department of Otorhinolaryngology-Head and Neck Surgery, National Medical Center, Seoul; \\ ${ }^{2}$ Department of Otolaryngology, Hanil General Hospital, Seoul; \\ ${ }^{3}$ Shimmian Rhinoplasty Clinic, Seoul; \\ Departments of ${ }^{4}$ Neurology and ${ }^{5}$ Otolaryngology, Asan Medical Center, University of Ulsan College of Medicine, Seoul, Korea
}

Objectives. To investigate the apnea-hypopnea index (AHI) according to the sleep stage in more detail after control of posture.

Methods. Patients who underwent nocturnal polysomnography between December 2007 and July 2018 were retrospectively evaluated. Inclusion criteria were as follows: age $>18$ years, sleep efficacy $>80 \%$, and patients who underwent polysomnography only in the supine position ( $100 \%$ of the time). Patients were classified into different groups according to the methods: the first, rapid eye movement (REM)-dominant group ( $\mathrm{AHI}_{\mathrm{REM}} / \mathrm{AHI}_{\mathrm{NREM}}>2$ ), non-rapid eye movement (NREM)-dominant group ( $\mathrm{AHI}_{\mathrm{NREM}} / \mathrm{AHI}_{\mathrm{REM}}>2$ ), and non-dominant group; and the second, light sleep group ( $\mathrm{AHI}_{\mathrm{N} 1 \mathrm{~N} 2}>\mathrm{AHI}$ sws) and slow wave sleep (SWS) group $\left(\mathrm{AHI}_{\mathrm{sws}}>\mathrm{AHI}_{\mathrm{N} 1 \mathrm{~N} 2}\right)$.

Results. A total of 234 patients (mean age, $47.4 \pm 13.9$ years) were included in the study. There were 108 patients (46.2\%) in the REM-dominant group, $88(37.6 \%)$ in the non-dominant group, and $38(16.2 \%)$ in the NREM-dominant group. The AHI was significantly higher in the NREM-dominant group than in the REM-dominant group (32.9 \pm 22.9 events/hr vs. $18.3 \pm 9.5$ events/hr, respectively). There were improvements in the AHI from stage 1 to SWS in NREM sleep with the highest level in REM sleep. A higher $\mathrm{AHI}_{\text {sws }}$ than $\mathrm{AHI}_{\mathrm{N} 1 \mathrm{~N} 2}$ was found in 16 of 234 patients (6.8\%); however, there were no significant predictors of these unexpected results except AHI.

Conclusion. Our results demonstrated the highest AHI during REM sleep stage in total participants after control of posture. However, there were $16.2 \%$ of patients showed NREM-dominant pattern $\left(\mathrm{AHI}_{\mathrm{NREM}} / \mathrm{AHI}_{\mathrm{REM}}>2\right)$ and $6.8 \%$ of patients showed higher $\mathrm{AHI}_{\mathrm{sws}}$ than $\mathrm{AHI}_{\mathrm{N} 1 \mathrm{~N} 2}$. Therefore, each group might have a different pathophysiology of obstructive sleep apnea (OSA), and we need to consider this point when we treat the patients with OSA.

Keywords. Obstructive Sleep Apnea; Sleep Stages; REM Sleep; Slow-Wave Sleep

\section{INTRODUCTION}

Obstructive sleep apnea (OSA), a common clinical disorder, is characterized by recurrent upper airway collapse and obstruc-

- Received June 18, 2018

Revised September 7, 2018

Accepted September 25, 2018

- Corresponding author: Yoo-Sam Chung

Department of Otolaryngology, Asan Medical Center, University of Ulsan College of Medicine, 88 Olympic-ro 43-gil, Songpa-gu, Seoul 05505, Korea Tel: +82-2-3010-3716, Fax: +82-2-489-2773

E-mail: yschung@amc.seoul.kr

*These authors contributed equally to this work. tion. Twenty-four percent of men and $9 \%$ of women experience at least five events of disordered breathing per hour of sleep [1]. The diagnosis and severity of OSA is typically reflected by the apnea-hypopnea index (AHI), which is a measure of the total number of total or partial upper airway obstructions lasting $>10$ seconds, divided by the sum of sleep time.

In clinical studies, OSA severity frequently changes over the course of a night according to the sleep stage. It has also been widely accepted that rapid eye movement (REM)-AHI may be higher than non-rapid eye movement (NREM)-AHI in OSA patients [2-7], and OSA would be more severe during light sleep compared with deep sleep stage.

Copyright (C) 2019 by Korean Society of Otorhinolaryngology-Head and Neck Surgery.

This is an open-access article distributed under the terms of the Creative Commons Attribution Non-Commercial License (http://creativecommons.org/licenses/by-nc/4.0)

which permits unrestricted non-commercial use, distribution, and reproduction in any medium, provided the original work is properly cited. 
However, there are some discrepancies in the AHI according to the sleep stage. Some studies have reported that the severity of OSA is higher during slow wave sleep (SWS) or NREM sleep compare to light sleep or REM sleep in patients with severe OSA [8-10]. But the studies published so far did not indicate proportions of the small group of patients and did not consider positional effect.

Therefore, the present study aimed to investigate the AHI according to the sleep stage (i.e., REM, NREM stage N1 and N2, and SWS) in more detail after control of posture, as well as to explore clinical variables affecting differences in the AHI according to the sleep stage if possible.

\section{MATERIALS AND METHODS}

\section{Study population}

The clinical and polysomnographic features of patients who underwent nocturnal polysomnography at the Asan Medical Center (Seoul, Korea) between December 2007 and July 2018 were retrospectively reviewed. Inclusion criteria were as follows: age at diagnosis $>18$ years, AHI $>5$ events/hr in polysomnography, and patients who underwent polysomnography in the supine position ( $100 \%$ of the time) were included to control for posture. Patients with sleep efficacy $\leq 80 \%$ and SWS $\leq 1$ minute were excluded. Finally, 234 patients were included in this study. This study was approved by the Institutional Review Board of Asan Medical Center (IRB No. 2018-1095). Informed consent was waived as this is a retrospective study.

\section{Subgroup analysis}

A commonly used diagnostic criterion is based on the ratio of AHI during REM sleep to the AHI during NREM sleep (AHI $\mathrm{AEM}_{\text {/ }}$ $\mathrm{AHI}_{\mathrm{NREM}}$ ), with a value of at least two indicating a predominance of disordered breathing during REM sleep. Following this criterion, the patients were divided into one of the three groups to compare clinical and polysomnographic features: REM-dominant $\left(\mathrm{AHI}_{\mathrm{REM}} / \mathrm{AHI}_{\text {NREM }}>2\right)$, NREM-dominant $\left(\mathrm{AHI}_{\mathrm{NREM}} / \mathrm{AHI}_{\mathrm{REM}}\right.$ $>2$ ), and non-dominant groups. $\mathrm{AHI}_{\mathrm{N} 1 \mathrm{N2}}$ (AHI of N1 and N2 sleep stage) was also calculated for the evaluation of light sleep stage. The equation for $\mathrm{AHI}_{\mathrm{N} 1 \mathrm{~N} 2}$ is as follows:

$\mathrm{AHI}_{\mathrm{N} 1 \mathrm{~N} 2}=\left(\mathrm{AHI}_{\mathrm{N} 1} \times \mathrm{Time}_{\mathrm{N} 1}+\mathrm{AHI}_{\mathrm{N} 2} \times \mathrm{Time}_{\mathrm{N} 2}\right) /\left(\mathrm{Time}_{\mathrm{N} 1}+\mathrm{Time}_{\mathrm{N} 2}\right)$

\section{H I G H L I G H T T S}

- Apnea-hypopnea index (AHI) was the highest during rapid eye movement sleep.

- AHI was significantly higher in the non-rapid eye movement sleep-dominant group.

- Higher AHI was found during deep sleep in 16 of 234 patients (6.8\%), differently from previous studies.
Patients were also divided into one of the two groups: light sleep group (with higher $\mathrm{AHI}_{\mathrm{N} 1 \mathrm{N2}}$ than $\mathrm{AHI}_{\text {sws }}$ ) and SWS group (with higher $\mathrm{AHI}_{\text {sws }}$ than $\mathrm{AHI}_{\mathrm{N1N2}}$ ). All patients were evaluated for the body mass index (BMI), age, sex, AHI, respiratory disturbance index (RDI), $\mathrm{AHI}_{\mathrm{NREM}}, \mathrm{AHI}_{\mathrm{N} 1}, \mathrm{AHI}_{\mathrm{N} 2}, \mathrm{AHI}_{\text {sws }}$, and $\mathrm{AHI}_{\mathrm{REM}}$.

\section{Statistical analysis}

All continuous quantitative variables are presented as mean and standard deviation, and normal distribution of variables was assessed using the Kolmogorov-Smirnov test. Comparisons between and among the REM-dominant, non-dominant, and NREM-dominant groups were performed using one-way analysis of variance (ANOVA). Categorical variables were assessed using Fisher exact test. For observations of the AHI according to the sleep stage (REM, NREM stage N1 and N2, SWS), statistical analysis was performed using repeated measures ANOVA and multiple comparison by Bonferroni correction. Comparisons between the

Table 1. Clinical and polysomnographic features of patients with obstructive sleep apnea

\begin{tabular}{lc}
\hline Variable & Value $(\mathrm{n}=234)$ \\
\hline Age (yr) & $47.4 \pm 13.9$ \\
Sex & $197(84.2)$ \\
Male & $37(15.8)$ \\
Female & $25.9 \pm 3.7$ \\
BMI (kg/m²) & $359.1 \pm 129.3$ \\
Total sleep time (min) & $30.3 \pm 19.4$ \\
RDI (events/hr) & $26.9 \pm 19.1$ \\
AHI (events/hr) & \\
NREM & $267.9 \pm 78.2$ \\
Time (min) & $24.5 \pm 20.5$ \\
AHI (events/hr) & $28.2 \pm 21.0$ \\
RDI (events/hr) & \\
N1 & $75.0 \pm 50.5$ \\
Time (min) & $39.4 \pm 25.1$ \\
AHI (events/hr) & $44.8 \pm 24.8$ \\
RDI (events/hr) & \\
N2 & $160.8 \pm 60.2$ \\
Time (min) & $20.0 \pm 18.7$ \\
AHI (events/hr) & $23.4 \pm 19.2$ \\
RDI (events/hr) & \\
SWS & $33.9 \pm 32.4$ \\
Time (min) & $10.6 \pm 18.2$ \\
AHI (events/hr) & $12.3 \pm 18.2$ \\
RDI (events/hr) & \\
REM & $55.8 \pm 29.4$ \\
Time (min) & $39.9 \pm 23.2$ \\
AHI (events/hr) & $40.8 \pm 22.5$ \\
RDI (events/hr) & \\
\hline & \\
VI & \\
\hline
\end{tabular}

Values are presented as mean \pm standard deviation or number (\%).

$\mathrm{BMI}$, body mass index; RDI, respiratory disturbance index; $\mathrm{AHI}$, apneahypopnea index; NREM, non-rapid eye movement; SWS, slow-wave sleep; REM, rapid eye movement. 
light sleep and SWS group were performed using independent sample $t$-tests and the Mann-Whitney $U$-tests for normally and non-normally distributed data, respectively. All analyses were performed using SPSS ver. 18.0 (SPSS Inc., Chicago, IL, USA). A $P<0.05$ was considered to be statistically significant.

\section{RESULTS}

\section{Clinical and polysomnographic characteristics of patients}

A total of 234 eligible patients (197 [84.2\%] men and 37 [15.8\%] women), with a median age of $47.4 \pm 13.9$ years were evaluated. Baseline demographics and polysomnographic data are summarized in Table 1. The mean total sleep time was $359.1 \pm 129.3$ minutes, with an REM sleep time of $55.8 \pm 29.4$ minutes and NREM sleep time of $267.9 \pm 78.2$ minutes. The mean AHI was $26.9 \pm 19.1$ events/hr and mean RDI was $30.3 \pm 19.4$ events/hr. In the entire sample, the mean $\mathrm{AHI}_{\mathrm{REM}}$ was higher than the mean AHI $_{\text {NREM }}(39.9 \pm 23.2$ events/hr vs. $24.5 \pm 20.5$ events/hr). Among the NREM sleep stage, the mean AHI tended to decrease with N1 (39.4 \pm 25.1 events/hr), N2 (20.0 \pm 18.7 events/ $\mathrm{hr})$, and SWS (10.6 \pm 18.2 events/hr) stage. Among all patients, there were statistically significant differences (overall $P<0.001$ ) in the AHI according to each sleep stage: $\mathrm{AHI}_{\mathrm{REM}}$ was higher than $\mathrm{AHI}_{\mathrm{N} 1}(P=1.000), \mathrm{AHI}_{\mathrm{N} 1}$ was higher than $\mathrm{AHI}_{\mathrm{N} 2}(P<0.001)$, and $\mathrm{AHI}_{\mathrm{N} 2}$ was higher than $\mathrm{AHI}_{\mathrm{sws}}(P=0.001)$ (Fig. 1).

\section{Comparison of REM-dominant, NREM-dominant, and non-dominant groups}

There were 108 patients (46.2\%) in the REM-dominant group, $88(37.6 \%)$ in the non-dominant group, and $38(16.2 \%)$ in the NREM-dominant group. Table 2 presents a comparison of the

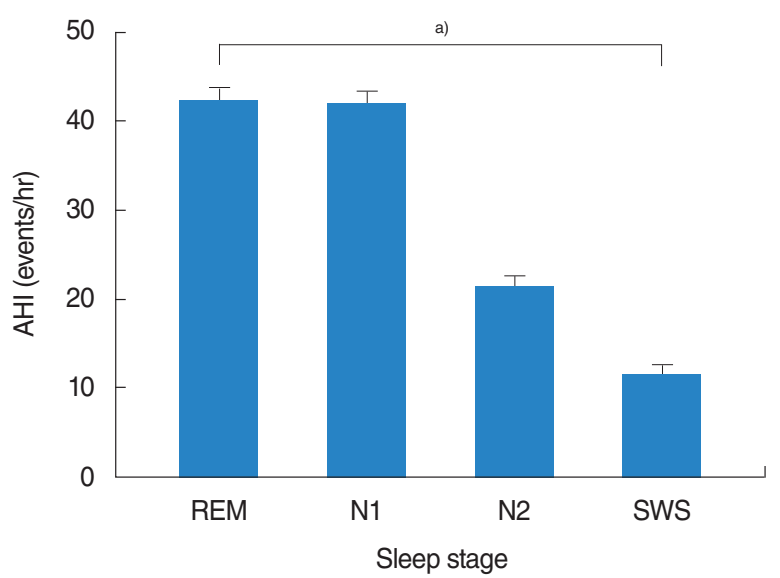

Fig. 1. Apnea-hypopnea index (AHI) according to sleep stage. REM, rapid eye movement; SWS, slow-wave sleep. ${ }^{a)}$ Repeated measures analysis of variance: overall $P<0.001$ (assessed using multiple comparison by Bonferroni's correction).

Table 2. Comparison of clinical and polysomnographic features among the REM-dominant, non-dominant, and NREM-dominant groups

\begin{tabular}{|c|c|c|c|c|}
\hline Variable & REM-dominant $(n=108)$ & Non-dominant $(\mathrm{n}=88)$ & NREM-dominant $(n=38)$ & $P$-value ${ }^{a)}$ \\
\hline Age (yr) & $45.5 \pm 12.9$ & $51.0 \pm 16.1$ & $48.2 \pm 13.8$ & 0.082 \\
\hline Sex (male:female) & $16: 4$ & $24: 5$ & $5: 1$ & $1.000^{b)}$ \\
\hline $\mathrm{BMI}\left(\mathrm{kg} / \mathrm{m}^{2}\right)$ & $26.2 \pm 3.6$ & $25.7 \pm 3.2$ & $25.5 \pm 3.9$ & 0.439 \\
\hline Total sleep time (min) & $322.5 \pm 103.0$ & $398.3 \pm 122.4$ & $372.5 \pm 178.1$ & $<0.001$ \\
\hline AHI (events/hr) & $18.3 \pm 9.5$ & $37.2 \pm 19.7$ & $32.9 \pm 22.9$ & $<0.001$ \\
\hline RDI (events/hr) & $21.9 \pm 9.8$ & $44.5 \pm 19.2$ & $34.5 \pm 23.2$ & $<0.001$ \\
\hline AHI $I_{\text {NREM }}$ (events/hr) & $13.1 \pm 8.8$ & $38.2 \pm 19.8$ & $32.6 \pm 23.4$ & $<0.001$ \\
\hline RDI $_{\text {NREM }}$ (events/hr) & $16.9 \pm 9.5$ & $46.5 \pm 19.3$ & $34.3 \pm 23.9$ & $<0.001$ \\
\hline Time $_{\text {NREM }}(\min )$ & $259.4 \pm 76.3$ & $276.6 \pm 74.1$ & $271.8 \pm 91.6$ & 0.291 \\
\hline $\mathrm{AHI}_{\mathrm{N} 1}$ (events/hr) & $28.8 \pm 19.9$ & $49.1 \pm 21.8$ & $48.3 \pm 27.2$ & $<0.001$ \\
\hline $\mathrm{RDI}_{\mathrm{N} 1}($ events/hr) & $35.0 \pm 19.7$ & $59.6 \pm 19.3$ & $50.5 \pm 27.7$ & $<0.001$ \\
\hline TimeN1 $_{\text {(min) }}$ & $56.1 \pm 32.7$ & $88.7 \pm 56.4$ & $97.2 \pm 59.1$ & $<0.001$ \\
\hline $\mathrm{AHI}_{\mathrm{N} 2}$ (events/hr) & $10.6 \pm 8.3$ & $33.0 \pm 20.6$ & $25.8 \pm 21.2$ & $<0.001$ \\
\hline $\mathrm{RDI}_{\mathrm{N} 2}$ (events/hr) & $14.2 \pm 9.4$ & $40.1 \pm 20.6$ & $27.4 \pm 21.3$ & $<0.001$ \\
\hline Time $_{\mathrm{N} 2}(\mathrm{~min})$ & $167.9 \pm 60.1$ & $159.7 \pm 61.0$ & $143.1 \pm 56.2$ & 0.090 \\
\hline $\mathrm{AHI}_{\text {sws }}$ (events/hr) & $3.3 \pm 4.9$ & $23.7 \pm 26.5$ & $13.8 \pm 20.1$ & $<0.001$ \\
\hline RDIsws (events/hr) & $4.7 \pm 5.3$ & $26.3 \pm 25.5$ & $15.5 \pm 20.3$ & $<0.001$ \\
\hline Timesws (min) & $37.9 \pm 34.2$ & $28.4 \pm 29.6$ & $35.5 \pm 32.4$ & 0.115 \\
\hline$A H I_{R E M}($ events/hr) & $45.7 \pm 21.8$ & $36.7 \pm 24.1$ & $34.3 \pm 22.9$ & 0.002 \\
\hline $\mathrm{RDI}_{\text {REM }}($ events/hr) & $47.7 \pm 20.8$ & $40.4 \pm 23.2$ & $35.2 \pm 22.5$ & $<0.001$ \\
\hline Timerem (min) & $60.3 \pm 31.6$ & $54.6 \pm 27.0$ & $45.9 \pm 26.2$ & 0.030 \\
\hline
\end{tabular}

Values are presented as mean \pm standard deviation.

REM, rapid eye movement; NREM, non-rapid eye movement; BMI, body mass index; AHI, apnea-hypopnea index; RDI, respiratory disturbance index; SWS, slow-wave sleep.

Assessed using ${ }^{\text {a) }}$ one-way analysis of variance except for ${ }^{\text {b) }}$ Fisher exact test. 
clinical and polysomnographic features of the three groups. The mean AHI was significantly higher in the NREM-dominant group than in the REM-dominant group $(32.9 \pm 22.9$ events/hr vs $18.3 \pm 9.5$ events/hr, respectively; $P<0.001)$. Among the NREM sleep stage, the AHI and RDI of N1, N2, and SWS were also significantly higher in the NREM-dominant group than in the REM-dominant group $(P<0.001$, respectively). The AHI and RDI of the REM sleep stage were significantly higher in the REM-dominant group than in the NREM-dominant group $(P=0.002$ and $P<0.001$, respectively). In the present study, no significant differences were observed among the three groups in age, BMI, or sex ( $P>0.05$ for all).

\section{Comparison of light sleep and deep sleep groups}

To analyze light sleep stage (N1 and N2) and deep sleep stage (SWS), AHI $_{\text {N1N2 }}$ was compared with AHIsws. Almost all patients had higher $\mathrm{AHI}_{\mathrm{N} 1 \mathrm{~N} 2}$ than $\mathrm{AHI}_{\text {sws; }}$, however, 16 of 234 (6.8\%) patients exhibited a higher $\mathrm{AHI}_{\text {sws }}$ than $\mathrm{AHI}_{\mathrm{N} 1 \mathrm{~N} 2}$. The clinical and polysomnographic characteristics of the two groups are summarized in Table 3. The data showed a higher AHI and RDI in the SWS group than in the light sleep group, even during SWS peri-

Table 3. Comparison of clinical and polysomnographic features between the light sleep and SWS groups

\begin{tabular}{|c|c|c|c|}
\hline Variable & $\begin{array}{c}\text { Light sleep group } \\
\left(A H_{N_{1 N 2}}>A H I_{\text {sws }} \text {, }\right. \\
n=218)\end{array}$ & $\begin{array}{c}\text { SWS group } \\
\left(A_{H} \|_{\text {N1N2 }}<A H I_{s w s},\right. \\
n=16)\end{array}$ & $P$-value \\
\hline Age (yr) & $47.2 \pm 14.0$ & $50.0 \pm 12.8$ & $0.438^{a)}$ \\
\hline Sex (male:female) & $182: 36$ & $15: 1$ & $0.277^{\mathrm{b})}$ \\
\hline BMl $\left(\mathrm{kg} / \mathrm{m}^{2}\right)$ & $25.9 \pm 3.7$ & $25.9 \pm 3.1$ & $0.951^{\text {a) }}$ \\
\hline Total sleep time (min) & $351.6 \pm 126.2$ & $461.5 \pm 132.9$ & $0.003^{c)}$ \\
\hline $\mathrm{AHI}$ (events/hr) & $26.0 \pm 18.7$ & $38.4 \pm 21.1$ & $0.326^{c)}$ \\
\hline RDI (events/hr) & $29.6 \pm 19.1$ & $40.2 \pm 21.2$ & $0.814^{c)}$ \\
\hline AHINREM (events/hr) & $23.5 \pm 20.0$ & $37.7 \pm 22.8$ & $0.016^{c)}$ \\
\hline $\mathrm{RDI}_{\text {NREM }}$ (events/hr) & $27.4 \pm 20.7$ & $39.6 \pm 22.9$ & $0.038^{c)}$ \\
\hline TimenREM $_{\text {NRin) }}$ & $266.0 \pm 79.0$ & $294.1 \pm 63.3$ & $0.177^{c)}$ \\
\hline $\mathrm{AH} \mathrm{I}_{\mathrm{N} 1}$ (events/hr) & $39.1 \pm 25.0$ & $44.0 \pm 27.7$ & $0.473^{c)}$ \\
\hline $\mathrm{RDI}_{\mathrm{N} 1}$ (events/hr) & $44.7 \pm 24.7$ & $46.3 \pm 27.6$ & $0.723^{c)}$ \\
\hline Time $_{\mathrm{N} 1}(\mathrm{~min})$ & $74.2 \pm 50.0$ & $86.0 \pm 57.4$ & $0.437^{c)}$ \\
\hline $\mathrm{AH} I_{\mathrm{N} 2}$ (events/hr) & $19.1 \pm 18.4$ & $32.1 \pm 19.0$ & $0.006^{c)}$ \\
\hline $\mathrm{RDI}_{\mathrm{N} 2}$ (events/hr) & $22.6 \pm 19.0$ & $33.6 \pm 18.9$ & $0.018^{c)}$ \\
\hline $\operatorname{Time}_{\mathrm{N} 2}(\mathrm{~min})$ & $160.1 \pm 60.9$ & $170.8 \pm 49.6$ & $0.492^{a)}$ \\
\hline AHIIsws (events/hr) & $7.8 \pm 13.8$ & $47.8 \pm 28.2$ & $<0.001^{c)}$ \\
\hline RDIsws (events/hr) & $9.6 \pm 14.2$ & $47.8 \pm 28.2$ & $<0.001^{\mathrm{c})}$ \\
\hline Timesws (min) & $34.2 \pm 31.8$ & $30.1 \pm 41.0$ & $0.323^{c)}$ \\
\hline AHIREM (events/hr) & $39.6 \pm 23.4$ & $44.4 \pm 19.7$ & $0.415^{c)}$ \\
\hline $\mathrm{RDI}_{\text {REM }}$ (events/hr) & $41.5 \pm 22.7$ & $45.6 \pm 19.7$ & $0.488^{a)}$ \\
\hline Time $_{\text {REM }}$ (min) & $55.6 \pm 29.4$ & $58.8 \pm 29.4$ & $0.674^{\mathrm{a})}$ \\
\hline
\end{tabular}

Values are presented as mean \pm standard deviation.

SWS, slow-wave sleep; AHI, apnea-hypopnea index; BMI, body mass index; RDI, respiratory disturbance index; NREM, non-rapid eye movement; REM, rapid eye movement.

Assessed using a)independent sample $t$-test, ${ }^{b}$ Fisher exact test, and ${ }^{c} M$ Mann-Whitney U-test. ods; the AHI of the SWS group was approximately seven times higher than that of the light sleep group. Significant differences were observed in AHI and RDI in NREM sleep stage, especially in N2 and SWS between the two groups. This results indicated that severe OSA patients exhibited more severe $\mathrm{AHI}_{\text {sws }}$ compared with mild to moderate OSA patients.

\section{DISCUSSION}

The activity of the respiratory muscles and upper airway occlusion varies according to sleep stage [3,11]. Among the various sleep stages, individuals are most vulnerable to disordered breathing - with a greater length of apneas, hypoxemia, and hypercapnia-in REM sleep than in any other sleep stage [5]. The tone of the upper airway, including the genioglossus, decreases from wakefulness to NREM sleep and is the lowest in REM sleep, predisposing to airway collapse [4,12]. Furthermore, medullary chemosensitivity to hypoxia and hypercapnia exhibit similar trends in the reduction of respiratory response during REM sleep $[13,14]$. Therefore, many OSA patients experience more respiratory events in REM sleep with the highest degree of oxygen desaturation [6]. The present findings demonstrated similar tendencies in that apnea occurred more often during the REM sleep stage in the total study population (Table 1).

In the NREM sleep stages, frequent arousal and apnea threshold according to sleep stage may contribute breathing instability. When arousal does occur, it enhances the hyperventilation following termination of the respiratory event and thus the arousal can yield hypocapnia below apnea threshold and further airway obstruction on return to sleep [15]. Among the NREM sleep stages, OSA improves during SWS. Ratnavadivel et al. [16] demonstrated an improvement in the AHI and arousal index from stage 1 to 4 NREM sleep $(P<0.001)$. This may be related to sleep stage-specific influences on dynamic upper airway control. It is well known that SWS is associated with increased upper airway dilator muscle activity [17]. Moreover, a relatively higher respiratory arousal threshold during SWS has been identified as a breathing stabilizing factor, affecting the improvement in apnea during SWS $[18,19]$. If the respiratory drive is elevated, breathing becomes unstable, leading to upper airway obstruction. In contrast, if the respiratory drive is low, as during SWS, breathing becomes stable. Our results also demonstrated the lowest AHI during SWS, followed by N1 and N2 sleep, and REM sleep, in that order.

The present study, however, yielded some novel findings after subgroup analysis. First, there were 38 (16.2\%) NREM-dominant patients who exhibited an $\mathrm{AHI}_{\mathrm{NREM}} \mathrm{AHI}_{\mathrm{NREM}}$ that was two times higher than $\mathrm{AHI}_{\text {REM }}$ (Table 2). In this study, we defined an NREM-dominant patient as similar to a REM-dominant individual; the subgroup analysis showed that the severity of the AHI in the NREM-dominant group was higher than that of the REM- 
dominant group. Although several other studies have reported results similar to ours [8-10], our findings are more meaningful because they are a simple comparison of the AHI in REM sleep and NREM sleep without any specific definition and control of posture. In future studies involving a larger sample size, it would be ideal to investigate the characteristics of these NREM-dominant patients if more can be recruited.

Second, several studies have recently compared the AHI according to the NREM sleep stage, and there have been reports that the AHI improved in SWS. In the present study, similar results were obtained in general, but there were an unexpected 16 patients $(6.8 \%)$ whose $\mathrm{AHI}_{\text {sws }}$ was higher than $\mathrm{AHI}_{\mathrm{N} 1 \mathrm{~N} 2}$. As shown in Table 3, there was some trend toward higher AHI in those patients, suggesting that OSA was more severe in patients in whom $\mathrm{AHI}_{\text {sws }}$ was higher than $\mathrm{AHI}_{\mathrm{N} 1 \mathrm{N2}}$.

One possible explanation for these unusual findings is the impairment of a compensatory mechanism(s). Healthy and OSA patients with intact neuromuscular compensatory mechanisms appear to compensate for an anatomically compromised upper airway through protective reflexes that increase upper airway dilator muscle activity to maintain airway patency during sleep [20]. For instance, the tone of the upper airway muscles, including the genioglossus, decreases from wakefulness to NREM sleep and is the lowest in REM sleep [4,12], and SWS is associated with increased upper airway dilator muscle activity that contributes to the improvement in apnea severity [17]. However, patients with severe OSA may have a sensory impairment of upper airway structures, which make them unable to respond to negative pharyngeal pressure due to repeated mechanical trauma and/or hypoxemia [21]. Therefore, in these patients, the compensatory mechanism mentioned above does not function properly; therefore, the AHI may become more severe in NREM and SWS. Further studies are needed to clarify the pathophysiology and explain these unusual findings.

The subgroup analysis, which represents one of the strengths of our study, revealed heterogenetic features of OSA. Considering this point, when we titrate the optimal pressure of continuous positive airway pressure, we may need to consider not only supine REM sleep, which is common, but also other sleep stages that have the highest AHI values. Another strength of our study is that we enrolled patients who underwent polysomnography in the supine position ( $100 \%$ of the time) at total sleep stage to control for posture because the collapsibility of the upper airway and the severity of OSA is strongly influenced by the body position $[22,23]$.

However, there are some limitations in this present study. The first is that the study sample was evaluated retrospectively. Second, we had not considered factors that might have influence on the sleep position. Severity of OSA and other factors may have influence on the sleep position. Further studies are needed to clarity the factors affecting the sleep position. Third, there is a selection bias because we choose the patients who underwent polysomnography only in the supine position $(100 \%$ of the time). If we did not select the patients in supine position $100 \%$, we should analyze subgroup in each sleep position and each sleep stage to adjust positional effect. Then the number of subgroup would be much larger and analysis would be too complicated. Therefore, we decided to control of posture to see more clearly the difference of AHI according to sleep stage.

Various factors, such as the BMI, sex, age, body position, and medication, are known to contribute to the development of OSA, and it can be presumed that these factors affect the quality and quantity of sleep by different mechanisms in each sleep stage. There are many treatment options for OSA, including weight loss, positional therapy, surgery, and positive airway pressure devices. The effect of treatment options on sleep apnea may differ within the sleep stage; moreover, suitable treatment options for each type of sleep-disordered breathing remains unclear. Further studies are needed to determine the significance of different sleep stages, and we need to take a different approach to each type of sleep-disordered breathing because each sleep stage may contribute to a different pathophysiology of OSA.

\section{CONFLICT OF INTEREST}

No potential conflict of interest relevant to this article was reported.

\section{ORCID}

Sung Hee Kim https://orcid.org/0000-0001-7230-8774

Yoo-Sam Chung https://orcid.org/0000-0002-8866-5415

\section{REFERENCES}

1. Young T, Palta M, Dempsey J, Skatrud J,Weber S, Badr S. The occurrence of sleep-disordered breathing among middle-aged adults. N Engl J Med. 1993 Apr;328(17):1230-5.

2. Eckert DJ, Malhotra A. Pathophysiology of adult obstructive sleep apnea. ProcAm Thorac Soc. 2008 Feb;5(2):144-53.

3. Horner RL. Motor control of the pharyngeal musculature and implications for the pathogenesis of obstructive sleep apnea. Sleep. 1996 Dec;19(10):827-53.

4. Wiegand L, Zwillich CW, Wiegand D, White DP. Changes in upper airway muscle activation and ventilation during phasic REM sleep in normal men. J Appl Physiol (1985). 1991 Aug;71(2):488-97.

5. Findley LJ, Wilhoit SC, Suratt PM. Apnea duration and hypoxemia during REM sleep in patients with obstructive sleep apnea. Chest. 1985 Apr;87(4):432-6.

6. Series F, Cormier Y, La Forge J. Influence of apnea type and sleep stage on nocturnal postapneic desaturation. Am Rev Respir Dis. 1990 Jun;141(6):1522-6.

7. Younes M. Contributions of upper airway mechanics and control mechanisms to severity of obstructive apnea. Am J Respir Crit Care 
Med. 2003 Sep;168(6):645-58.

8. Muraki M, Kitaguchi S, Ichihashi H, Haraguchi R, Iwanaga T, Kubo $\mathrm{H}$, et al. Apnoea-hypopnoea index during rapid eye movement and non-rapid eye movement sleep in obstructive sleep apnoea. J Int Med Res. 2008 Sep-Oct;36(5):906-13.

9. Liu Y, Su C, Liu R, Lei G, Zhang W, Yang T, et al. NREM-AHI greater than REM-AHI versus REM-AHI greater than NREM-AHI in patients with obstructive sleep apnea: clinical and polysomnographic features. Sleep Breath. 2011 Sep;15(3):463-70.

10. Siddiqui F, Walters AS, Goldstein D, Lahey M, Desai H. Half of patients with obstructive sleep apnea have a higher NREM AHI than REMAHI. Sleep Med. 2006 Apr;7(3):281-5.

11. Remmers JE, deGroot WJ, Sauerland EK, Anch AM. Pathogenesis of upper airway occlusion during sleep. J Appl Physiol Respir Environ Exerc Physiol. 1978 Jun;44(6):931-8.

12. Katz ES, White DP. Genioglossus activity during sleep in normal control subjects and children with obstructive sleep apnea. Am J Respir Crit Care Med. 2004 Sep;170(5):553-60.

13. Douglas NJ, White DP, Weil JV, Pickett CK, Martin RJ, Hudgel DW, et al. Hypoxic ventilatory response decreases during sleep in normal men.Am Rev Respir Dis. 1982 Mar;125(3):286-9.

14. Douglas NJ, White DP,Weil JV, Pickett CK, Zwillich CW. Hypercapnic ventilatory response in sleeping adults. Am Rev Respir Dis. 1982 Nov;126(5):758-62.

15. Eckert DJ, Malhotra A, Jordan AS. Mechanisms of apnea. Prog Cardiovasc Dis. 2009 Jan-Feb;51(4):313-23.
16. Ratnavadivel R, Chau N, Stadler D, Yeo A, McEvoy RD, Catcheside PG. Marked reduction in obstructive sleep apnea severity in slow wave sleep. J Clin Sleep Med. 2009 Dec;5(6):519-24.

17. Pillar G, Fogel RB, Malhotra A, Beauregard J, Edwards JK, Shea SA, et al. Genioglossal inspiratory activation: central respiratory vs mechanoreceptive influences. Respir Physiol. 2001 Aug;127(1):23-38.

18. Ratnavadivel R, Stadler D, Windler S, Bradley J, Paul D, McEvoy RD, et al. Upper airway function and arousability to ventilatory challenge in slow wave versus stage 2 sleep in obstructive sleep apnoea. Thorax. 2010 Feb;65(2):107-12.

19. Saboisky J, Eckert D, Malhotra A. Stable breathing through deeper sleeping.Thorax. 2010 Feb;65(2):95-6.

20. Mezzanotte WS, Tangel DJ, White DP. Waking genioglossal electromyogram in sleep apnea patients versus normal controls (a neuromuscular compensatory mechanism). J Clin Invest. 1992 May;89(5): 1571-9.

21. Petrof BJ, Hendricks JC, Pack AI. Does upper airway muscle injury trigger a vicious cycle in obstructive sleep apnea? A hypothesis. Sleep. 1996 Jul;19(6):465-71.

22. Lee SA, Paek JH, Chung YS, Kim WS. Clinical features in patients with positional obstructive sleep apnea according to its subtypes. Sleep Breath. 2017 Mar;21(1):109-17.

23. Penzel T, Moller M, Becker HF, Knaack L, Peter JH. Effect of sleep position and sleep stage on the collapsibility of the upper airways in patients with sleep apnea. Sleep. 2001 Feb;24(1):90-5. 